\title{
Scavenging rate constants of hydrophilic antioxidants against multiple reactive oxygen species
}

\author{
Yoshimi Sueishi, ${ }^{1, *}$ Masashi Hori, ${ }^{1}$ Misa Ishikawa, ${ }^{1}$ Keiko Matsu-ura, ${ }^{1}$ Erisa Kamogawa, ${ }^{1}$ Yusaku Honda, ${ }^{1}$ \\ Masakazu Kita ${ }^{2}$ and Keishi Ohara ${ }^{3}$ \\ ${ }^{1}$ Department of Chemistry, Faculty of Science, Okayama University and ${ }^{2}$ Graduate School of Education, Okayama University, \\ 3-1-1 Tsushima-naka, Kita-ku, Okayama 700-8530, Japan \\ ${ }^{3}$ Graduate School of Science and Engineering, Ehime University, Matsuyama 790-8577, Japan
}

(Received 2 July, 2013; Accepted 23 September, 2013; Published online 21 February, 2014)

\begin{abstract}
Scavenging rate constants of eight hydrophilic antioxidants, including caffeic acid, chlorogenic acid, genistein, glutathione, $\boldsymbol{N}$ acetylcysteine, rutin, trolox, and uric acid against multiple ROS, namely superoxide anion, hydroxyl radical, singlet oxygen, and alkoxyl radical were determined with the electron spin resonance method. Direct flash photolysis measurement of the second-order rate constant in the reaction of alkoxyl radical plus the spin trap 5,5-dimethyl-pyrroline $\mathrm{N}$-oxide made it possible to evaluate scavenging rate constants in antioxidants. The magnitudes of scavenging rate constants were notably dependent on the character of each ROS and the overall rate constants were highest in hydroxyl radical scavenging and the lowest in superoxide anion. The highest scavenging rate constant against superoxide anion was obtained by chlorogenic acid $\left(2.9 \times 10^{5} \mathrm{M}^{-1} \mathrm{~s}^{-1}\right)$ and the lowest was by $\mathrm{N}$-acetylcysteine $\left(5.0 \times 10^{2} \mathrm{M}^{-1} \mathrm{~s}^{-1}\right)$. For singlet oxygen, the highest was by glutathione $\left(9.4 \times 10^{8} \mathrm{M}^{-1} \mathrm{~s}^{-1}\right)$ and the lowest was by uric acid $\left(2.3 \times 10^{6} \mathrm{M}^{-1} \mathrm{~s}^{-1}\right)$. All other numbers are listed and illustrated. Redox potential measurements of the antioxidants indicated that the antioxidants are likely to react with superoxide anion and singlet oxygen through electron transfer processes.
\end{abstract}

Key Words: reactive oxygen species (ROS), hydrophilic antioxidant, radical scavenging, spin trapping, redox potential

$\mathrm{R}$ eactive oxygen species (ROS) such as superoxide anion $\left(\mathrm{O}_{2}{ }^{-\cdot}\right)$, hydroxyl radical $\left(\mathrm{HO}^{*}\right)$, and singlet oxygen $\left({ }^{1} \mathrm{O}_{2}\right)$ are produced as a result of biochemical processes in vivo, and are considered as main causes of oxidative damage in cells and tissues. ${ }^{(1,2)}$ Oxidative damage in biological systems is essentially the oxidation of lipids and proteins and can be inhibited or delayed by antioxidants $^{(3)}$; therefore, scavenging rate determination of antioxidants against ROS is meaningful. Previous studies evaluated the oxygen radical scavenging capacity (ORSC) of pure antioxidant compounds and foods. ${ }^{(4-6)}$ Conventional ORSC method utilizes oxidative damage of certain fluorescent probe, but has a few experimental disadvantages. ${ }^{(6)}$ We proposed a new method of ORSC evaluation (ORSC-ESR or ORSC-EPR), using an electron spin resonance (ESR) trapping technique. ${ }^{(7,8)}$ Thus, we have reported ORSC-ESR measurement of the scavenging rates in various lipophilic antioxidant compounds. ${ }^{(9)}$ However, the ORSC method uses a single free radical species that is generated from the azo-initiator 2,2'-azobis(2-amidino-propane) dihydrochloride (AAPH). This free radical species has been identified as alkoxyl radical ( $\left.\mathrm{RO}^{*}\right)$. $^{(10)}$

Antioxidants possess scavenging ability against multiple ROS. The scavenging rate certainly depends on the kind of ROS to be scavenged, while a majority of previous studies determined scavenging rates against a single ROS. Recently, Oowada et al. ${ }^{(11)}$ proposed a method to determine scavenging capacity against multiple ROS in biological specimens such as human serum. ORSC-ESR method has been also utilized to evaluate antioxidant capacity of food such as mushrooms. ${ }^{(12)}$ However, ROS scavenging data for pure antioxidant compounds are still lacking. In this study, we selected eight well-documented pure antioxidant compounds and generated data sets of relative scavenging rate constants against superoxide anion, hydroxyl radical and singlet oxygen.

Furthermore, the scavenging rate constant between the spin trap DMPO and $\mathrm{RO}^{*}$ was directly evaluated with flash photolysis technique. By combining this rate constant with relative rate constants from ESR measurement, all scavenging rate constants were calculated. In addition, in order to verify the mechanism of scavenging reaction we performed redox potential measurements for the antioxidants.

\section{Materials and Methods}

Materials and equipment. The spin traps used are shown in Fig. 1 ; i.e., 5,5-dimethyl-pyrroline $\mathrm{N}$-oxide (DMPO) and 5-(2,2dimethyl-1,3-propoxy cyclophosphoranyl)-5-methyl-1-pyrroline $N$-oxide (CYPMPO). These compounds were purchased from Tokyo Chemical Industry Co., Ltd. (Tokyo, Japan) and Radical Research Inc. (Hino, Japan), respectively. 2,2,6,6-Tetramethyl-4piperidone hydrochloride (TMPD) was obtained from Aldrich Chemical Company Inc. (Milwaukee, WI) and used as the detection reagent of singlet oxygen. ${ }^{(13,14)}$ Rose bengal and riboflavin were obtained from Wako Pure Chem. Ind., Ltd. (Osaka, Japan) and used as photo-sensitizers to generate singlet oxygen and superoxide anion, respectively. Hydrogen peroxide and AAPH were purchased from Wako Pure Chem. and was used as a source of hydroxyl and alkoxyl radicals, respectively. Fig. 1 shows the eight natural hydrophilic antioxidants used in this study. Trolox, reduced glutathione, caffeic acid (3,4-dihydroxycinnamic acid), rutin, chlorogenic acid, and $N$-acetylcysteine (NAC) were obtained from Wako Pure Chem. Genistein and uric acid were purchased from Tokyo Chemical Industry and Nacalai Tesque, Inc. (Kyoto, Japan), respectively. Water was purified by distillation, and a $100 \mathrm{mM}\left(\mathrm{M}=\mathrm{mol} \mathrm{dm}{ }^{-3}\right)$ sodium phosphate buffer (pH 7.4) was used as a solvent.

A JEOL FA200 X-band spectrometer (Akishima, Japan) was

*To whom correspondence should be addressed.

E-mail: ysueishi@okayama-u.ac.jp 
<smiles></smiles>

DMPO<smiles></smiles>

CYPMPO<smiles>CC1(C)CC(=O)CC(C)(Cl)N1</smiles>

TMPD

\section{Antioxidants}<smiles>Cc1c(C)c2c(c(C)c1O)CCC(C)(C(=O)O)O2</smiles>

Trolox<smiles>O=C(O)C=Cc1ccc(O)c(O)c1</smiles>

Caffeic acid<smiles>O=c1c(-c2ccc(O)cc2)coc2cc(O)cc(O)c12</smiles>

Genistein<smiles>O=C(/C=C/c1ccc(O)c(O)c1)OC1CC(O)(C(=O)O)CC(O)C1O</smiles>

Chlorogenic acid<smiles>N[C@@H](CCC(=O)N[C@@H](CS)C(=O)NCC(=O)O)C(=O)O</smiles>

Glutathione<smiles>O=c1[nH]c(=O)c2[nH]c(=O)[nH]c2[nH]1</smiles>

Uric acid<smiles>CC(=O)N[C@@H](CS)C(=O)O</smiles>

NAC

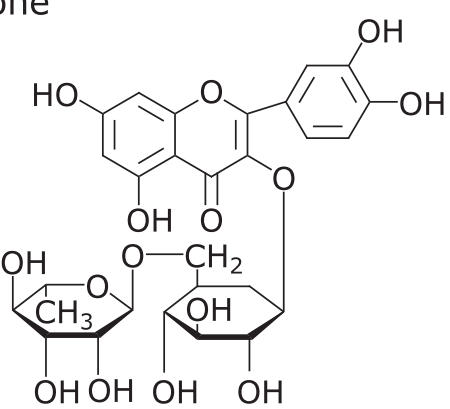

Rutin

Fig. 1. Structures of DMPO, CYPMPO, TMPD and various hydrophilic antioxidants.

used to record ESR spectra. Spectrometer settings were: sweep time of $30 \mathrm{~s}$, time constant of $0.1 \mathrm{~s}$, and microwave power of $5 \mathrm{~mW}$. The ESR signal intensities of the spin adduct in the presence and absence of antioxidants were measured to calculate the scavenging rate constants according to the kinetic equation shown in the following section.

Scavenging of superoxide anion. The ESR spin trapping method was applied to evaluate free radical scavenging rates. The spin-trapping method has been recognized as a useful tool for the detection and identification of unstable free radicals. ${ }^{(15)}$ Spin trapping compounds (spin traps) such as CYPMPO react with superoxide to form stable free radical called spin adducts (Scheme 1).<smiles>CCON1C([O-])CCC1(OC(=O)[O-])P1(=O)CCC(C)(C)CO1</smiles>

Scheme 1
ESR signal pattern was used to identify the spin adduct and its intensity was used for the kinetic measurements of superoxide anion. The superoxide anion was generated from riboflavin $(60 \mu \mathrm{M})$ and ethylendiaminetetraacetic acid disodium salt dehydrate (EDTA, $10 \mathrm{mM}$ ) with $30 \mathrm{~s}$ UV irradiation. UV source was a $200 \mathrm{~W}$ mercury arc with a band-path $(500-600 \mathrm{~nm})$ filter (RUF-203S, Radical Research Inc., Hino, Japan). After UV irradiation, the ESR signal of the spin adducts with CYPMPO $(10 \mathrm{mM})$ was recorded (Fig. 2a).

Kinetic formulation of the competitive reaction between the spin trap CYPMPO and antioxidant has been published elsewhere. ${ }^{(7)}$ Briefly, in the presence of antioxidants (AOx) and the spin trap CYPMPO, the superoxide anion scavenging reaction should occur as follows:

$\mathrm{O}_{2}+$ Riboflavin $\stackrel{\mathrm{hv}}{\longrightarrow} \mathrm{O}_{2}^{-\cdot}$

$\mathrm{AOX}+\mathrm{O}_{2}^{-\cdot} \longrightarrow$ Products, $k_{\mathrm{AOx}}$

$\mathrm{ST}(\mathrm{CYPMPO})+\mathrm{O}_{2}{ }^{-\bullet} \longrightarrow$ Spin adduct $\left(\mathrm{CYPMPO}-\mathrm{O}_{2}^{-\bullet}\right), k_{\mathrm{ST}}$

where $\mathrm{ST}$ denotes the spin trap reagent. $k_{\mathrm{AOx}}$ and $k_{\mathrm{ST}}$ are rate 

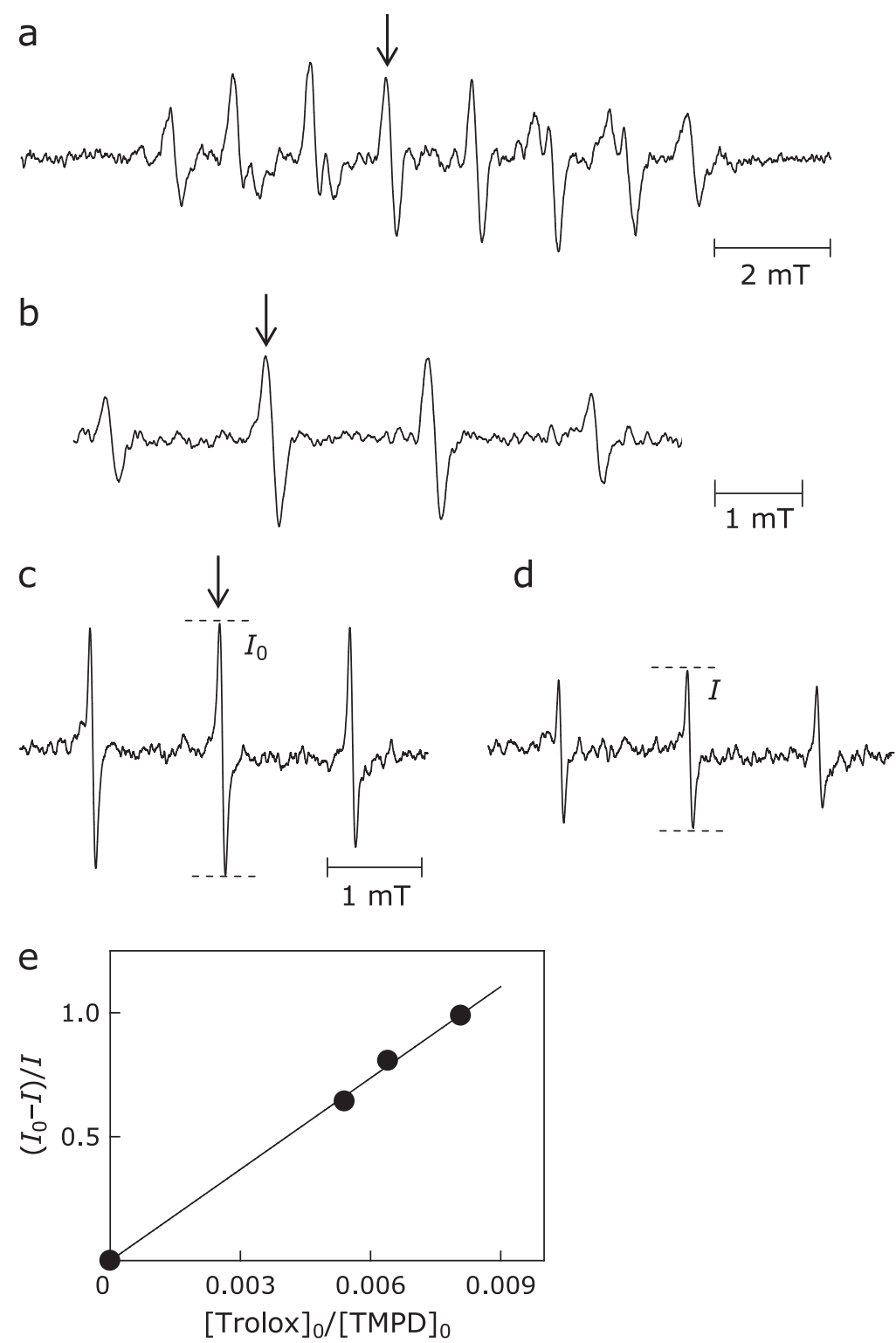

Fig. 2. ESR spectra of spin adducts produced after photolysis of precursors/sensitizers: (a) Superoxide anion adduct of CYPMPO (Isomer 1: $A_{\mathrm{P}}=5.3 \mathrm{mT}, A_{\mathrm{N}}=1.29 \mathrm{mT}, A_{H}=1.15 \mathrm{mT}$; Isomer $\left.2: A_{\mathrm{P}}=5.10 \mathrm{mT}, A_{N}=1.31 \mathrm{mT}, A_{H}=1.03 \mathrm{mT}\right)$. (b) Hydroxyl radical adduct of $\mathrm{DMPO}\left(A_{\mathrm{N}}=A_{H}=\right.$ $1.50 \mathrm{mT}$ ). ( $c$ and $d)$ Tempol radical formed after the reaction of singlet oxygen with TMPD $\left(A_{N}=1.60 \mathrm{mT}\right)$ : $[$ trolox] $0=(c) 0$ and $(d) 480 \mu M$. $[T M P D]_{0}=100 \mathrm{mM}$. Horizontal broken lines in the ESR spectra demonstrate the change in signal height by the addition of the antioxidant trolox. (e) A plot of $\left(I_{0}-1\right) / I$ vs [trolox] $/[\text { TMPD] }]_{0}$ according to Eq. (1). Peaks marked with arrows were used for the antioxidant capacity assay.

constants. A simple formula for the superoxide scavenging capacity calculation can be derived from the above reactions: ${ }^{(7)}$

$\frac{\mathrm{I}_{0}-\mathrm{I}}{\mathrm{I}}=\frac{k_{\mathrm{AOx}}[\mathrm{AOx}]_{0}}{k_{\mathrm{ST}}[\mathrm{ST}]_{0}}$

where I and I0 are ESR signal heights of the spin adduct in the presence and absence of antioxidants, respectively. The symbol [ ]o denotes the initial concentration of the component. The relative superoxide scavenging rate was determined from the slope $\left(k_{\mathrm{AOx}} / k_{\mathrm{ST}}\right)$ of a plot of $\left(\mathrm{I}_{0}-\mathrm{I}\right) / \mathrm{I}$ against $[\mathrm{AOx}]_{0} /[\mathrm{ST}]_{0}$. The hydrophilic antioxidant trolox has been conventionally adopted as a standard. ${ }^{(6)}$ The relative superoxide scavenging rate is expressed as ORSC values in the trolox equivalent unit (TEU).

Hydroxyl radical and alkoxyl radical scavenging. The spin trap DMPO was employed for hydroxyl radical and alkoxyl radical because DMPO provides unique ESR spectral patterns for these radicals and the stability of the spin adducts is excellent (Scheme 2).

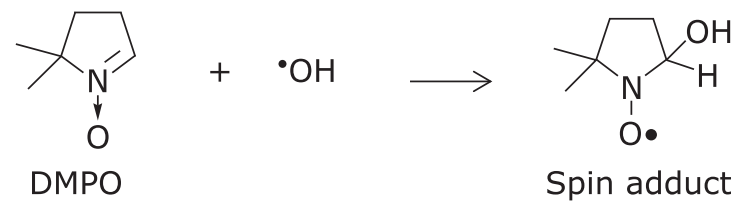

Scheme 2

Hydroxyl radical was generated by UV irradiation of a $\mathrm{H}_{2} \mathrm{O}_{2}$ solution $(4 \mathrm{mM})$ and the UV photolysis of AAPH was adopted to produce alkoxyl radical. After UV irradiation, the ESR signal of 
the hydroxyl radical adduct with DMPO $(5 \mathrm{mM})$ was recorded by an ESR spectrometer (Fig. 2b). Equation 1 for the hydroxyl radical scavenging assay was formulated using the competitive reaction system described below: ${ }^{(7)}$

$$
\begin{aligned}
& \mathrm{H}_{2} \mathrm{O}_{2} \stackrel{\mathrm{hv}}{\longrightarrow} 2 \mathrm{HO}^{*} \text { or AAPH } \stackrel{\text { hv }}{\longrightarrow} \mathrm{RO}^{*} \\
& \mathrm{AOx}+\mathrm{R}^{\cdot} \longrightarrow \text { Products, } k_{\mathrm{AOx}} \\
& \mathrm{DMPO}+\mathrm{R}^{\cdot} \longrightarrow \text { DMPO-R, } k_{\mathrm{ST}}
\end{aligned}
$$

where $\mathrm{R}^{*}$ represents either $\mathrm{HO}^{*}$ or $\mathrm{RO}^{*}$. Kinetic formulation to calculate relative scavenging rate constants is exactly the same as Eq. (1), but ST should be equal to DMPO in this case.

Singlet oxygen scavenging. The reaction between singlet oxygen and TMPD to form a stable nitroxide radical was utilized to quantify singlet oxygen (Scheme 3$).^{(13,14)}$

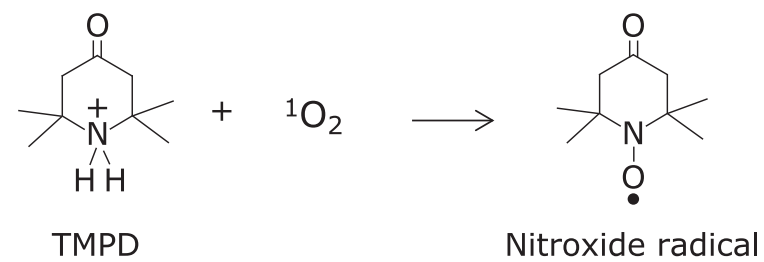

Scheme 3

Exactly speaking, this reaction is not classified as spin trapping, but for the sake of convenience we treat this as spin trapping reaction.

Singlet oxygen was generated via VIS irradiation $(5 \mathrm{~s}$, bandpath of $500-600 \mathrm{~nm})$ of aqueous rose bengal solution $(30 \mu \mathrm{M})$. In the presence of TMPD and antioxidants, the following competitive reaction should occur:

$$
\mathrm{O}_{2}+\text { Rose bengal } \stackrel{\mathrm{hv}}{\longrightarrow}{ }^{1} \mathrm{O}_{2}
$$

$\mathrm{AOx}+{ }^{1} \mathrm{O}_{2} \longrightarrow$ Products, $k_{\mathrm{AOx}}$

$\mathrm{TMPD}+{ }^{1} \mathrm{O}_{2} \longrightarrow$ Nitroxide radical, $k_{\mathrm{TMPD}}$

As shown in Fig. 2c and d, when TMPD and AOx (e.g., trolox) were mixed, the ESR peak intensity decreased, compared with that in the absence of AOx, indicating that part of ${ }^{1} \mathrm{O}_{2}$ was scavenged or deactivated by the antioxidant. According to the above reactions, the relative ${ }^{1} \mathrm{O}_{2}$ scavenging rates can be evaluated from Eq. (1) for ESR signal intensity. A typical plot of $\left(\mathrm{I}_{0}-\mathrm{I}\right) / \mathrm{I}$ against $[\text { trolox }]_{0} /[\mathrm{TMPD}]_{0}$ for the trolox/TMPD system is shown in Fig. 2e with a straight line passing through the origin, suggesting that the above competitive mechanism is justifiable.

Flash photolysis determination of $\mathrm{RO}^{*}$ trapping rate constant. A laser flash photolysis system (TSP-1000, UNISOKU, Hirakata, Japan) equipped with a Nd-YAG laser (Continuum Surelight-I, FWHM $<5 \mathrm{~ns}, 2 \mathrm{~Hz}$ ) was employed to determine the trapping rate constant of DMPO against $\mathrm{RO}^{\bullet}$ radical. Excitation light source $\left[\lambda_{\text {ex }}\right.$ (excitation wavelength) $=355 \mathrm{~nm}$ ] was used to generate $\mathrm{RO}^{*}$ radical from AAPH in a phosphate buffer $(0.1 \mathrm{M})$.

The RO• adduct of DMPO has one absorption band at $260 \mathrm{~nm}$ and time course of this band was followed after the flash photolysis. The reaction mechanism for the AAPH decomposition leading to the formation of ROO ${ }^{\circ}$ and $\mathrm{RO}^{\circ}$ is well documented. ${ }^{(16,17)}$ In excess DMPO, the pseudo-first-order kinetics would be held for the DMPO trapping reaction, thus the reaction mechanism for the DMPO-OR adduct formation can be expressed as follows:

$$
\begin{aligned}
& \mathrm{AAPH} \stackrel{\mathrm{h} v}{\longrightarrow} \mathrm{R}^{\cdot} \stackrel{\mathrm{O}_{2}}{\longrightarrow} \mathrm{ROO}^{*} \\
& 2 \mathrm{ROO}^{*} \longrightarrow 2 \mathrm{RO}^{*}+\mathrm{O}_{2}, k_{1} \\
& \mathrm{RO}^{*}+\mathrm{DMPO}^{\longrightarrow} \mathrm{DMPO}-\mathrm{OR}, k_{2}\left(\mathrm{RO}^{\cdot} \longrightarrow \text { DMPO-OR, } k_{\mathrm{obs}}\right)
\end{aligned}
$$

where $k_{\text {obs }}\left(=k_{2}[\mathrm{DMPO}]_{0}\right)$ denotes the pseudo-first-order rate constant.

The kinetic formulation for the above reaction has been made ${ }^{(18)}$ and the concentration of DMPO-OR can be expressed as:

$$
\frac{[\mathrm{C}]}{[\mathrm{A}]_{0}}=1-\frac{1}{\omega}-e^{-a \omega}\left[e^{a}-\frac{e^{a \omega}}{\omega}+a\left\{\log \omega+a(\omega-1)+\frac{a^{2}}{4}\left(\omega^{2}-1\right)\right\}\right]
$$$$
a=\frac{k_{\mathrm{obs}}}{2[\mathrm{~A}]_{0} k_{1}}, \quad \omega=1+2[\mathrm{~A}]_{0} k_{1} t
$$

where $[\mathrm{C}]$ and $[\mathrm{A}]$ denote the concentrations of the DMPO-OR and ROO'. Appropriate curve fitting was performed for the time course curve and $k_{\text {obs }}$ was calculated according to Eq. (2).

Redox potential measurements of various antioxidants. An RRDE-3A rotating ring disk electrode (BAS) and an electrochemical analyzer model $701 \mathrm{D}(\mathrm{BAS})$ were used to measure the electrochemical properties (redox potentials) of antioxidants in a phosphate buffer at $298 \pm 1 \mathrm{~K}$ with $\mathrm{pH}=7.4,[\mathrm{AOx}]=1.0 \times$ $10^{-3} \mathrm{M}$, and $\left[\mathrm{NaClO}_{4}\right]=1 \mathrm{M}$. A glassy carbon rotating disk $(2,000 \mathrm{rpm})$ electrode, platinum wire electrode, and $\mathrm{Ag} / \mathrm{Ag}^{+}$electrode were used as the working, counter, and reference electrodes, respectively. The oxidation wave of $\mathrm{K}_{3} \mathrm{Fe}(\mathrm{CN})_{6}$ was observed at $+210 \mathrm{mV}$ vs $\mathrm{Ag} / \mathrm{Ag}^{+}$.

\section{Results and Discussion}

ESR spin trapping determination of relative scavenging rates. Table 1 lists the relative scavenging rate constants of eight hydrophilic antioxidants for superoxide anion, hydroxyl

\begin{tabular}{|c|c|c|c|c|c|c|c|c|c|}
\hline \multirow{2}{*}{ Antioxidants } & \multicolumn{2}{|c|}{$\mathrm{O}_{2}^{-\bullet}$} & \multicolumn{2}{|c|}{$\mathrm{HO}^{\circ}$} & \multicolumn{2}{|c|}{${ }^{1} \mathrm{O}_{2}$} & \multirow{2}{*}{$\begin{array}{l}\text { RO' } \\
\text { TEU }\end{array}$} & \multirow{2}{*}{$\begin{array}{c}E_{1 / 2}(\mathrm{Ox}) \\
(\mathrm{eV})\end{array}$} & \multirow{2}{*}{$\begin{array}{c}E-E_{1 / 2}(\mathrm{Ox}) \\
(\mathrm{eV})\end{array}$} \\
\hline & $k_{\mathrm{AOX}} / k_{\mathrm{CYPMPO}}$ & TEU & $k_{\text {AOX }} / k_{\text {DMPO }}$ & TEU & $k_{\text {AOX }} / k_{\text {TMPD }}$ & TEU & & & \\
\hline Chlorogenic acid & $5,980 \pm 319$ & $137 \pm 9$ & $3.43 \pm 0.03$ & $0.39 \pm 0.01$ & $758 \pm 11$ & $6.2 \pm 0.2$ & 2.3 & 0.36 & 3.45 \\
\hline Uric acid & $222 \pm 8$ & $5.1 \pm 0.7$ & $3.40 \pm 0.04$ & $0.38 \pm 0.01$ & $4.57 \pm 0.50$ & $0.037 \pm 0.004$ & $2.2^{*}$ & 0.47 & 3.79 \\
\hline Caffeic acid & $4,780 \pm 219$ & $109 \pm 6$ & $2.88 \pm 0.01$ & $0.33 \pm 0.01$ & $1,080 \pm 25$ & $8.8 \pm 0.3$ & $1.6^{*}$ & 0.31 & 3.68 \\
\hline Rutin & $322 \pm 12$ & $7.3 \pm 0.4$ & $2.28 \pm 0.05$ & $0.26 \pm 0.01$ & $685 \pm 11$ & $5.6 \pm 0.2$ & $1.4^{*}$ & 0.236 & 3.19 \\
\hline Trolox & $43.8 \pm 1.5$ & 1 & $8.85 \pm 0.03$ & 1 & $123 \pm 3$ & 1 & 1 & 0.23 & 4.08 \\
\hline Glutathione & $23.3 \pm 0.8$ & $0.53 \pm 0.01$ & $5.87 \pm 0.01$ & $0.66 \pm 0.01$ & $1,856 \pm 14$ & $15.1 \pm 0.3$ & $0.57^{*}$ & -0.01 & 6.06 \\
\hline Genistein & $57.3 \pm 2.0$ & $1.3 \pm 0.6$ & $4.23 \pm 0.05$ & $0.48 \pm 0.01$ & $384 \pm 15$ & $3.1 \pm 0.2$ & $0.43^{*}$ & 0.195 & 3.64 \\
\hline NAC & $10.3 \pm 0.4$ & $0.24 \pm 0.01$ & $4.87 \pm 0.03$ & $0.55 \pm 0.01$ & $404 \pm 6$ & $3.3 \pm 0.1$ & 0.40 * & $<-0.010$ & $>6.06$ \\
\hline
\end{tabular}
radical, and singlet oxygen. In the same table, relative scavenging rate constants were expressed in the trolox equivalent unit (TEU). Trolox is a vitamin $\mathrm{E}$ analog that has been employed as a standard antioxidant in the ORSC method, where ORSC values are expressed relative to those of trolox, i.e., trolox equivalent unit. ${ }^{(6,7)}$

Relative scavenging rates against $\mathrm{RO}^{\bullet}$ radical (ORSC values)

Table 1. Relative scavenging rate constants and values in TEU for ROS scavenging of various hydrophilic antioxidants

*Cited from Ref. (7). 

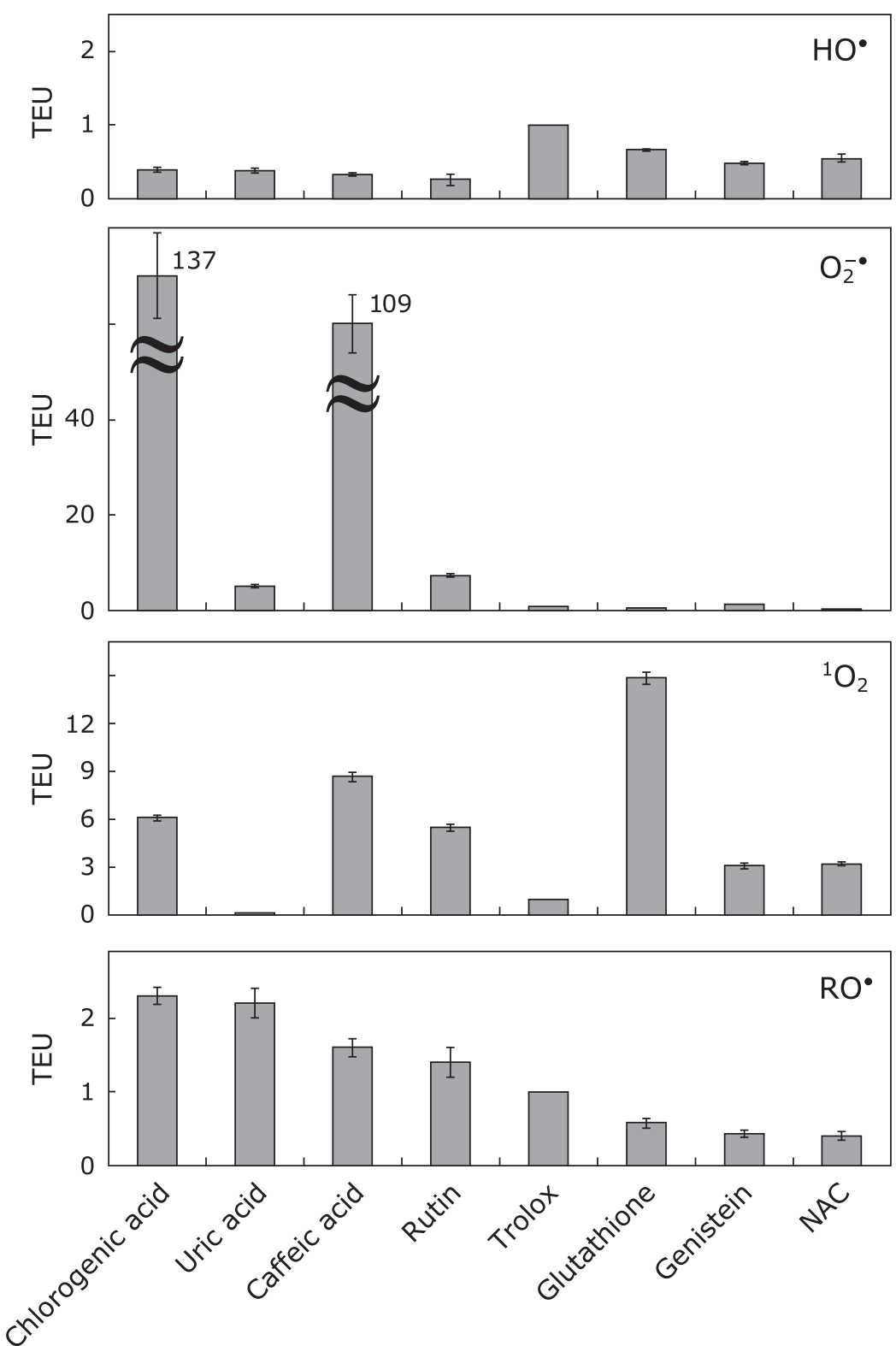

Fig. 3. A bar graph that represents the relative ROS scavenging abilities (TEU) for various hydrophilic antioxidants: $\left(\mathrm{HO}^{\circ}\right) \mathrm{hydroxyl}^{\circ} \mathrm{rdical}\left(\mathrm{O}_{2^{-}}\right)$ superoxide anion, $\left({ }^{1} \mathrm{O}_{2}\right)$ singlet oxygen, and (RO') AAPH-derived alkoxyl radical.

are also given in Table 1. Results indicated that: (1) superoxide anion: the magnitude in TEU ranged from 0.24 to 137 , and chlorogenic acid and caffeic acid showed the highest scavenging rates, and the order of superoxide scavenging rate is; chlorogenic acid $>$ caffeic acid $>$ rutin $>$ uric acid $>$ genistein $\approx$ trolox $>$ glutathione $>$ NAC, (2) hydroxyl radical: the magnitude in TEU ranged from 0.26 to 1 , and the rates are not sensitive to the structure of antioxidants, and the order of antioxidant ability is trolox $>$ glutathione $>$ genistein $>$ chlorogenic acid $\approx$ uric acid $\approx$ caffeic acid $\approx$ rutin, and (3) singlet oxygen: the magnitude in TEU ranged from 0.037 to 15.1 , and glutathione shows the highest antioxidant capacity, and the order of the rates is glutathione $>$ caffeic acid $>$ chlorogenic acid $\approx$ rutin $>$ NAC $\approx$ genistein $>$ trolox $>$ uric acid. The order is not in agreement with the data for superoxide anion. The magnitude in TEU can be compared only within the same ROS.

Fig. 3 shows the relative ROS scavenging rates of the eight hydrophilic antioxidants. These are relative values as compared with the relative $\mathrm{RO}^{*}$ radical scavenging rates (ORSC-ESR values). Also, Fig. 3 clearly indicates that the present relative ROS scavenging rates do not correlate with the relative $\mathrm{RO}^{*}$ radical scavenging rate.

Evaluation of DMPO trapping rate constants against RO. The rate constant for DMPO + $\mathrm{RO}^{*}$ reaction was measured using laser flash photolysis method. Fig. 4a shows the time dependence $\left([\mathrm{C}] /[\mathrm{A}]_{0}\right)$ of the DMPO-OR product. Using the kinetic parameters $\left(k_{1}=1.7 \times 10^{4} \mathrm{M}^{-1} \mathrm{~s}^{-1}\right)^{(16,17)}$ in Eq. (2), the $k_{\text {obs }}$ and $[\mathrm{A}]_{0}$ values were determined from fitting to the experimental data: $[\mathrm{A}]_{0}=1.0 \times 10^{-5} \mathrm{M}$ and $k_{\mathrm{obs}}=0.64,0.68$, and $0.92 \mathrm{~s}^{-1}$ at $[\mathrm{DMPO}]_{0}=$ $0.50,0.55$, and $0.70 \mathrm{mM}$, respectively.

The second order rate constant $k_{2}$ for the DMPO trapping reaction was evaluated to be $k_{2}=1.27 \times 10^{3} \mathrm{M}^{-1} \mathrm{~s}^{-1}$ from the slope of the plots of $k_{\text {obs }}$ against [DMPO] $]_{0}$ (Fig. 4b). A straight line that passes through the origin was obtained, indicating that the reaction 

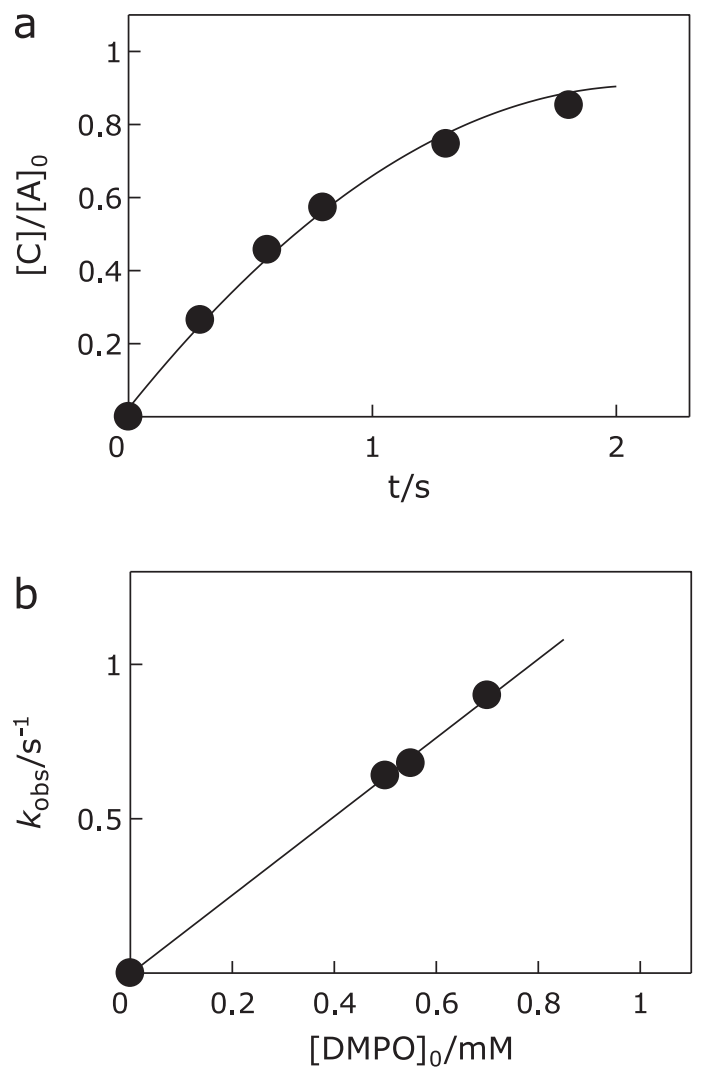

Fig. 4. (a) Time course curve of the DMPO-OR adduct formation. [C] is the adduct concentration under the conditions of: [DMPO] $0=0.5 \mathrm{mM}$, $[A A P H]_{0}=10 \mathrm{mM}$, and $[A]_{0}=10 \mu \mathrm{M}$. Solid line represents the $[C] /[A]_{0}$ values calculated from Eq. (2). (b) Plots of the $k_{\text {obs }}$ values against [DMPO]. scheme and calculation procedures using Eq. (2) are justifiable.

Direct measurement of the reaction rate constant has made it possible to directly compare the scavenging rate constants in multiple ROS. In the present experiment, using a competitive trapping method, we have determined the relative RO* spin trapping rates for CYPMPO and DMPO to be $k_{\mathrm{CYPMPO}} / k_{\mathrm{DMPO}}=17.8$, from which the rate constant of the $\mathrm{RO}^{*}+\mathrm{CYPMPO}^{-}$reaction was calculated to be $k_{\text {CYPMPO }}\left(\mathrm{RO}^{*}\right)=2.26 \times 10^{4} \mathrm{M}^{-1} \mathrm{~s}^{-1}$. The rate constants of traps (DMPO and CYPMPO) for $\mathrm{HO}^{*}$ and $\mathrm{O}_{2}{ }^{-} \cdot$ were determined to be $k_{\mathrm{DMPO}}\left(\mathrm{HO}^{*}\right)=2.8 \times 10^{9} \mathrm{M}^{-1} \mathrm{~s}^{-1}$ and $k_{\mathrm{CYPMPO}}\left(\mathrm{O}_{2}^{-\cdot}\right)=$ $48 \mathrm{M}^{-1} \mathrm{~s}^{-1}$, respectively. ${ }^{(19,20)}$ Rate constant data for ${ }^{1} \mathrm{O}_{2}$ scavenging was not available in the present experiments; therefore, we utilized the rate constants $\left[k_{\text {trolox }}\left({ }^{1} \mathrm{O}_{2}\right)=6.22 \times 10^{7} \mathrm{M}^{-1} \mathrm{~s}^{-1}\right.$ (21) $]$ between trolox and ${ }^{1} \mathrm{O}_{2}$ to calculate the rate constants for other antioxidants. Rate constant data are listed in Table 2 and these numbers are illustrated in a logarithmic scale (Fig. 5).

It should be pointed out that scavenging rate constants against $\mathrm{HO}^{*}$ radical is insensitive to the structure of antioxidants. The magnitude of the $\mathrm{HO}^{*}$ rate constants is close to diffusion control, suggesting that $\mathrm{HO}^{*}$ may react with any group of initial encounter. We also show that antioxidants scavenging rates against superoxide anion, hydroxyl radical, and singlet oxygen does not correlate with that for AAPH-derived RO' radical (ORSC value), indicating that ORSC value may not be representative antioxidant parameter. The logarithmic plot of the antioxidant's reaction rates in Fig. 5 indicates that the ROS scavenging rates increase in the order of $\mathrm{O}_{2}{ }^{-*}<\mathrm{RO}^{*}<{ }^{1} \mathrm{O}_{2}<\mathrm{HO}$.

Reaction mechanism of ROS scavenging reaction. Based on the redox potentials of the antioxidants, the mechanism of the antioxidant's reaction with ROS was elucidated. It is generally difficult to determine the half-wave potential values $\left(E_{1 / 2}\right)$ of redox reactions for phenolic antioxidants because of the potential window and follow-up chemical reaction. The rotating ring-disk electrode (RRDE) is a powerful technique for detecting the

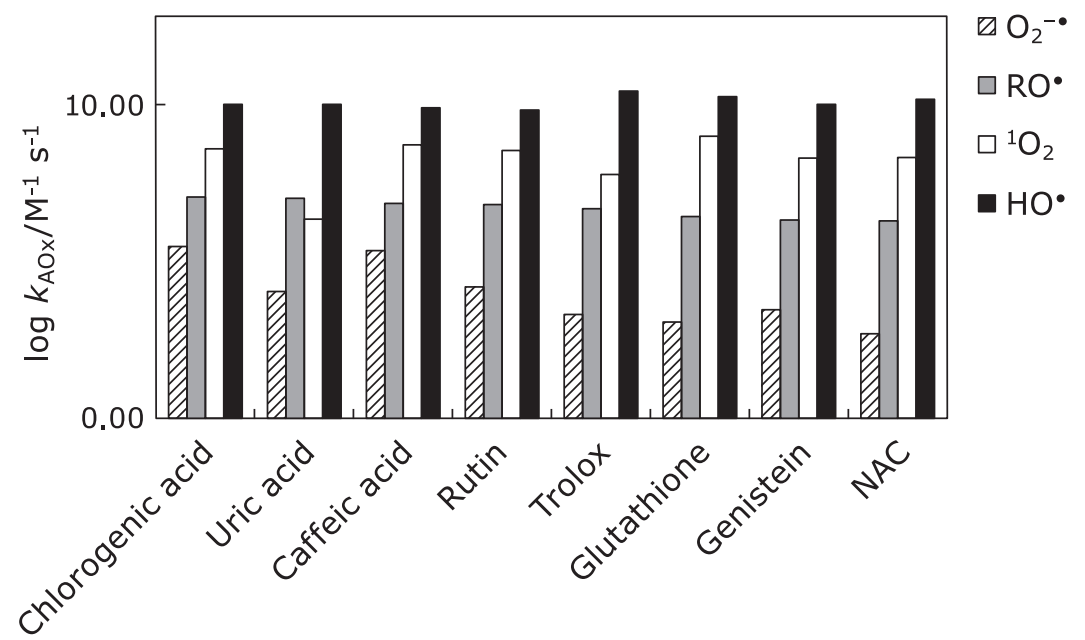

Fig. 5. A logarithmic representation of scavenging rate constants against various ROS in eight hydrophilic antioxidants.

Table 2. Rate constants $\left(k_{\mathrm{AOX}} / \mathrm{M}^{-1} \mathrm{~s}^{-1}\right)$ for ROS scavenging of various hydrophilic antioxidants

\begin{tabular}{lcclc}
\hline Antioxidants & \multicolumn{1}{c}{$\mathrm{O}_{2^{\bullet}}$} & \multicolumn{1}{c}{$\mathrm{HO}^{\bullet}$} & \multicolumn{1}{c}{$\mathrm{O}_{2}$} & \multicolumn{1}{c}{$\mathrm{RO}^{\bullet}$} \\
\hline Chlorogenic acid & $(288 \pm 15) \times 10^{3}$ & $(9.60 \pm 0.08) \times 10^{9}$ & $(38.6 \pm 0.5) \times 10^{7}$ & $(111 \pm 7) \times 10^{5}$ \\
Uric acid & $(10.6 \pm 0.4) \times 10^{3}$ & $(9.52 \pm 0.11) \times 10^{9}$ & $(2.30 \pm 0.25) \times 10^{6}$ & $(106 \pm 6) \times 10^{5}$ \\
Caffeic acid & $(229 \pm 10) \times 10^{3}$ & $(8.06 \pm 0.03) \times 10^{9}$ & $(54.7 \pm 1.2) \times 10^{7}$ & $(75.9 \pm 0.9) \times 10^{5}$ \\
Rutin & $(15.4 \pm 0.6) \times 10^{3}$ & $(6.38 \pm 0.14) \times 10^{9}$ & $(34.8 \pm 0.5) \times 10^{7}$ & $(69.0 \pm 8.5) \times 10^{5}$ \\
Trolox & $(2.10 \pm 0.07) \times 10^{3}$ & $(24.8 \pm 0.1) \times 10^{9}$ & $(6.22 \pm 0.15) \times 10^{7}$ & $(48.3 \pm 3.7) \times 10^{5}$ \\
Glutathione & $(1.11 \pm 0.04) \times 10^{3}$ & $(16.4 \pm 0.1) \times 10^{9}$ & $(93.9 \pm 0.7) \times 10^{7}$ & $(27.6 \pm 1.5) \times 10^{5}$ \\
Genistein & $(2.75 \pm 0.10) \times 10^{3}$ & $(11.8 \pm 0.2) \times 10^{9}$ & $(19.1 \pm 0.7) \times 10^{7}$ & $(20.7 \pm 1.6) \times 10^{5}$ \\
NAC & $(0.504 \pm 0.020) \times 10^{3}$ & $(13.6 \pm 0.1) \times 10^{9}$ & $(20.5 \pm 0.3) \times 10^{7}$ & $(19.3 \pm 2.5) \times 10^{5}$ \\
\hline
\end{tabular}


products, side-products or even short-lived intermediates of electrode reactions. ${ }^{(22)} \mathrm{We}$ have determined the $E_{1 / 2}(\mathrm{ox})$ values of antioxidants from the RRDE measurements. Fig. 6a shows the representative voltammogram of disk current in RRDE measurement for caffeic acid. As shown in Fig. 6a, the $E_{1 / 2}(\mathrm{ox})$ value of caffeic acid was determined with the aid of an attached computer program (an electrochemical analyzer model $701 \mathrm{D}$ ) and the data of antioxidants are listed in Table 1. The antioxidants' capacity for electron donation and acceptance can be expressed in terms of $E_{1 / 2}(\mathrm{ox})$ and $\Delta E-E_{1 / 2}(\mathrm{ox})$, respectively, where $\Delta E$ is the transition energy obtained from the absorption band in the UV region. ${ }^{(22,23)}$ Smaller values of $E_{1 / 2}(\mathrm{ox})$ mean easier electron transfer from the antioxidant. In contrast, the antioxidants having a small value of $\Delta E-E_{1 / 2}($ ox $)$ are good electron acceptors.

Fig. $6 \mathrm{~b}$ shows that the superoxide anion scavenging rates increase with the decrease in the $\Delta E-E_{1 / 2}$ (ox) value in antioxidants, suggesting that the primary process in the reaction of superoxide anion with antioxidants is characterized as reduction of antioxidants as follows ${ }^{(24)}$ :

$$
\mathrm{O}_{2}{ }^{-\cdot}+\mathrm{AOx} \longrightarrow \mathrm{O}_{2}+\text { reduced } \mathrm{AOx} \longrightarrow
$$

For the singlet oxygen scavenging, as shown in Fig. 6c, the antioxidant that has smaller $E_{1 / 2}(\mathrm{ox})$ shows higher scavenging rates, suggesting that the antioxidants were operative as electron donors, that is in agreement with the previous results reported by Foley et al. ${ }^{(25)}$ and Mukai et al. ${ }^{(26)}$

\section{Conclusion}

In eight hydrophilic antioxidants, we obtained scavenging rate constants against four ROS. The antioxidant that has high scavenging rate constant does not necessarily mean that it exercises high antioxidant activity in vivo because scavenging capacity (scavenging rate) value is calculated by multiplying the rate constant with the antioxidant concentration. It is highly likely that the local concentration of antioxidant varies a great deal in vivo. Therefore, one should be very cautious in judging the effectiveness of the antioxidant.

\section{Acknowledgments}

We thank Dr. Yashige Kotake for helpful discussion and critical reading of the manuscript. This work was supported in part by a Grant-in-Aid for Science Research (C) (No. 25450169) from the Ministry of Education, Culture, Sports, Science, and Technology of Japan.

\section{Abbreviations}

$\begin{array}{ll}\text { AAPH } & \text { 2,2'-azobis(2-amidinopropane) dihydrochloride } \\ \text { AOx } & \text { antioxidant } \\ \text { CYPMPO } & \text { 5-(2,2-dimethyl-1,3-propoxy cyclophosphoranyl)- } \\ & \text { 5-methyl-1-pyrroline } N \text {-oxide } \\ \text { DMPO } & \text { 5,5-dimethyl-pyrroline } N \text {-oxide } \\ \text { ESR } & \text { electron spin resonance } \\ \text { NAC } & N \text {-acetylcysteine } \\ \text { ORSC } & \text { oxygen radical scavenging capacity } \\ \text { ORAC-ESR } & \text { ESR spin trapping-based ORAC method } \\ \text { RO } & \text { alkoxyl radical } \\ \text { ROS } & \text { reactive oxygen species }\end{array}$

\section{References}

1 Foley S, Navaratnam S, McGarvey DJ, Land EJ, Truscott TG, Rice-Evans A. Singlet oxygen quenching and redox properties of hydroxycinnamic acids. Free Radic Biol Med 1999; 26: 1202-1208.

2 Jiménez A, Romojaro F, Gómez JM, Llanos MR, Sevilla F. Antioxidant
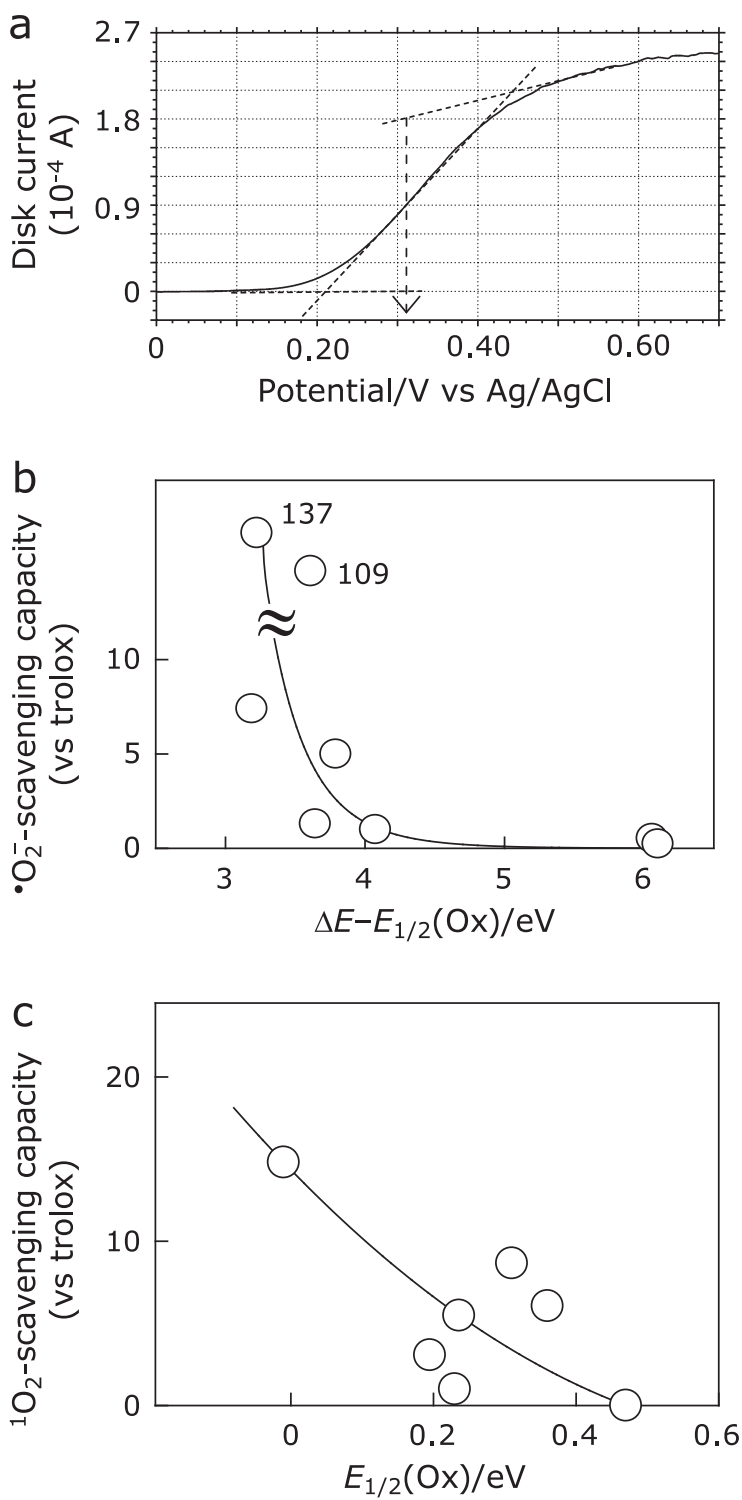

Fig. 6. Electrochemical properties of the eight antioxidants: (a) Voltammogram of disk current in RRDE measurement $(2,000 \mathrm{rpm})$ for caffeic acid. (b) The plot of superoxide anion scavenging rate constants against $\Delta E-E_{1 / 2}$ (ox) of each antioxidant. (c) The plot of singlet oxygen scavenging rate constants against the oxidation potential $E_{1 / 2}(\mathrm{ox})$ of each antioxidant.

$\begin{array}{ll}\text { ST } & \text { spin trap agent } \\ \text { TEU } & \text { trolox equivalent unit } \\ \text { TMPD } & \text { 2,2,6,6-tetramethyl-4-piperidone hydrochloride } \\ \text { trolox } & \begin{array}{l}\text { 6-hydroxy-2,5,7,8-tetra-methylchroman-2- } \\ \text { carboxylic acid } \\ \text { visible light }\end{array} \\ \text { VIS } & \end{array}$

\section{Conflict of Interest}

No potential conflicts of interest were disclosed.

systems and their relationship with the response of pepper fruits to storage at $20^{\circ}$ C. J Agric Food Chem 2003; 51: 6293-6299.

3 Wang SY, Chen H, Ehlenfeldt MK. Antioxidant capacities vary substantially among cultivars of rabbiteye blueberry (Vaccinium ashei Reade). Int J Food 
Sci Technol 2011; 46: 2482-2490.

4 Glazer AN. Phycoerythrin fluorescence-based assay for reactive oxygen species. Methods Enzymol 1990; 186: 161-168.

5 Cao G, Alessio HM, Cutler RG. Oxygen-radical absorbance capacity assay for antioxidants. Free Rad Biol Med 1993; 14: 303-311.

6 Huang D, Ou B, Hampsch-Woodill M, Flanagan JA, Prior RL. Highthroughput assay of oxygen radical absorbance capacity (ORAC) using a multichannel liquid handling system coupled with a microplate fluorescence reader in 96-well format. J Agric Food Chem 2002; 50: 4437-4444.

7 Kohri S, Fujii H, Oowada S, et al. An oxygen radical absorbance capacitylike assay that directly quantifies the antioxidant's scavenging capacity against AAPH-derived free radicals. Anal Biochem 2009; 386: 167-171.

8 Sueishi Y, Ishikawa M, Yoshioka D, et al. Oxygen radical absorbance capacity (ORAC) of cyclodextrin-solubilized flavonoids, resveratrol and astaxanthin as measured with the ORAC-EPR method. $J$ Clin Biochem Nutr 2012; 50: 127-132.

9 Ishikawa M, Sueishi Y, Endo N, et al. Cyclodextrin encapsulation of the functional group diminishes antioxidant's free radical scavenging rates. Int J Chem Kinet 2012; 44: 598-603.

10 Sueishi Y, Yoshioka D, Oowada S, et al. Is the oxygen radical absorbance capacity (ORAC) method a peroxyl-radical scavenging assay? $Z$ Phys Chem 2010; 224: 921-928.

11 Oowada S, Endo N, Kameya H, Shimmei M, Kotake Y. Multiple free-radical scavenging capacity in serum. J Clin Biochem Nutr 2012; 51: 117-121.

12 Kanno T, Kawamura S, Harada E, Kameya H, Ukai M, Osawa T. Radical scavenging ability by ESR spin trapping and oxygen radical absorbance capacity of hot water extracts from mushrooms. Nippon Shokuhin Kagaku Kaishi 2013; 60: 173-178.

13 Rabek JF, Ranby B, Arct J, Liu R. Singlet oxygen and free-radical oxidation of polydienes and related problems with stabilization: synergistic and antagonistic effects. J Photochem 1984; 25: 519-536.

14 Tokuoka Y, Niitsu A, Watabe N, Murakami TN, Kawashima N. ESR spectroscopy of singlet oxygen generated by protoporphyrin IX in aqueous surfactant solutions. J Oleo Sci 2003; 52: 135-140.

15 Janzen EG. Spin trapping. Methods Enzymol 1984; 105: 188-198.
16 Rojas Wahl RU, Zeng L, Madison SA, DePinto RL, Shay BJ. Mechanistic studies on the decomposition of water soluble azo-radical-initiators. J Chem Soc Perkin Trans 2 1998; 2009-2018.

17 Krainev AG, Bigelow DJ. Comparison of 2,2'-azobis(2-amidinopropane) hydrochloride (AAPH) and 2,2'-azobis(2,4-dimethylvaleronitrile) (AMVN) as free radical initiators: a spin-trapping study. J Chem Soc Perkin Trans 2 1996; 747-754.

18 Capellos C, Bielski BHJ. Kinetic Systems: Mathematical Description of Chemical Kinetics in Solution. John Wiley \& Sons Ltd., New York and London, 1972.

19 Taniguchi H, Madden KP. An in situ radiolysis time-resolved ESR study of the kinetics of spin trapping by 5,5-dimethyl-1-pyrroline- $N$-oxide. $J$ Am Chem Soc 1999; 121: 11875-11879.

20 Kamibayashi M, Oowada S, Kameda H, et al. Synthesis and characterization of a practically better DEPMPO-type spin trap, 5-(2,2-dimethyl-1,3-propoxy cyclophosphoryl)-5-methyl-1-pyrroline $N$-oxide (CYPMPO). Free Rad Res 2006; 40: 1166-1172.

21 Ohara K, Kikuchi K, Origuchi T, Nagaoka S. Singlet oxygen quenching by trolox C in aqueous micelle solutions. J Photochem Photobiol B 2009; 97: 132-137.

22 Okuno M, Kita M, Kashiwabara K, Fujita J. Redox potentials of cobalt(III) mixed-ligand complexes with sulfur, phosphorus, and nitrogen donor atoms and a correlation with their electronic spectra. Chem Lett 1989; 18: 16431646.

23 Dodsworth ES, Lever ABP. Correlations between electrochemical potentials and optical charge transfer energies in ruthenium bipyridine derivatives. Chem Phys Lett 1986; 124: 152-158.

24 Galano A, Vargas R, Martínez A. Carotenoids can act as anitioxidants by oxidizing the superoxide radical anion. Phys Chem Chem Phys 2010; 12: 193-200.

25 Foyer CH, Lelandais M, Kunert KJ. Photooxidative stress in plants. Physiol Plant 1994; 92: 696-717.

26 Mukai K, Nagai S, Ohara K. Kinetic study of the quenching reaction of singlet oxygen by tea catechins in ethanol solution. Free Radic Biol Med 2005; 39: 752-761. 